\title{
Marketplace or reselling? A signalling model
}

\author{
Nada Belhadj ${ }^{\text {a, }}$, Didier Laussel ${ }^{b}$, Joana Resende ${ }^{c}$ \\ a ISG, University of Tunis, Tunisia \\ ${ }^{\mathrm{b}}$ Aix-Marseille Univ, CNRS, EHESS, Centrale Marseille, AMSE, France \\ ${ }^{c}$ Cef. Up, Economics Department, University of Porto, Portugal
}

\begin{abstract}
:
This paper shows that the platforms' private information on demand may explain the empirical obser- vation that platforms like Amazon resell highdemand products, while acting as marketplace for low- demand goods. More precisely, the paper examines the strategic interaction between a seller and a bet- ter informed platform within a signalling game. We consider that the platform may choose between two distinct business models: act as a reseller or work as a pure marketplace between the buyers and the seller. The marketplace mode, which allows to internalize the spillover between the platform's sales and the seller's direct sales is always preferred for a low-value good. The reselling mode, which allows the platform to take advantage of its private information, may be selected in the case of high-value goods provided that (i) the externalities between direct sales and platform sales are not too strong and (ii) the difference between consumers' willingness to pay for the high and the low-value goods is large enough. Under these conditions, the game displays a Least-Cost Separating Equilibrium in which the platform works as a marketplace for low-demand goods, while it acts as a reseller in the case of high-demand goods.
\end{abstract}

JEL classification: $D 4, L 1$

Keywords: Marketplace, Reselling, Asymmetric information, Platform, Demand uncertainty

\section{Introduction}

In the last years, many economic sectors have experienced considerable changes. While in some cases there has been a significant disintermediation process, in other cases new intermediation players have emerged. Digital platforms are a good example of this last phenomenon (e.g. Amazon, Ebay or Alibaba). These platforms have adopted different types of business models: some of them act as resellers (retailers), whereas others are pure marketplaces, which bring together sellers and buyers (without making any sales decisions). Finally, in some other cases, digital platforms act both as a marketplace (for some products) and as a reseller (for other products). For example, Rysman (2009) reports that the Internet giant Amazon sometimes acts as a conventional reseller (Amazon Retail), while on other occasions it offers a marketplace to independent buyers and sellers (Amazon Marketplace). For some particular

\footnotetext{
in This article is a result of the project NORTE-01-0145-FEDER-028540, supported by Norte Portugal Regional Operational Programme (NORTE 2020), under the PORTUGAL 2020 Partnership Agreement, through the European Regional Development Fund (ERDF) and through national funds by the FCT - Fundação para a Ciência e a Tecnologia. This research also benefited from the support of COMPETE 2020 (POCI) and FCT through the project POCI-01-0145-FEDER-006890.

* Corresponding author.

E-mail addresses: nadabelhadj@gmail.com (N. Belhadj),

didier.laussel@univ-amu.fr (D. Laussel),

jresende@fep.up.pt (J. Resende).
}

products (e.g. specific books), Amazon basically buys the product at a wholesale price and then resells it. However, for many other products, ${ }^{1}$ Amazon adopts a two-sided business model, acting as a platform which connects buyers and sellers, with sellers choosing both the sales level and the price of the product.

Jiang et al. (2011) provided significant empirical evidence that platforms like Amazon tend to resell high-demand products while letting third-party independent sellers sell low-demand goods (with the platform opting for the marketplace mode). For example, in the category of electronics, they noticed that Amazon was reselling only $7 \%$ of the products but that those products included $64 \%$ of the 100 best sellers. In the categories of Tools and Home improvement, Sports and Outdoors, Jewelry, Toys and Games and Shoes the respective figures were $5.8 \%$ and $88 \%, 3.1 \%$ and $76 \%, 3.2 \%$ and $34 \%, 5.9 \%$ and $66 \%, 16.7 \%$ and $72 \% .^{2}$ These findings have been confirmed by Zhu and Liu (2016) who concluded from a large scale empirical study using data from Amazon.com that "the likelihood of Amazon's entry is positively correlated with the popularity and customer ratings of third-party sellers' products".

\footnotetext{
${ }^{1}$ Many of these products may have low individual sales but, collectively, if the distribution channel is large enough, they achieve a market share which exceeds the relatively few best sellers. This corresponds to the "long tail" of Internet sales, according to the term coined by Anderson (2006).

2 Table 1, page 758 in Jiang et al. (2011).
} 
The present paper proposes a very stylized model to examine whether asymmetric information on demand may constitute one possible determinant explaining why the platform may prefer to resell a high-value good, while acting as a marketplace for lowvalue goods.

To this end, we analyze the signalling game played between an intermediary platform and a representative seller (thereafter "she"), who may be interested in using the platform to distribute its product. More precisely, we assume that the seller may use (either separately or together) two distribution channels ${ }^{3}$ to reach its final consumers: (i) its own direct sales channel, such as a brick and mortar shop or an online website; (ii) an independent platform. One may find plethoric evidence of the combination of these business practices. For example, HP computers are for sale on HP online store as well as on Amazon and other platforms, Nike shoes may be purchased on Nike official site as well as on Amazon, Dior perfumes are available on Dior's official site as well as on many intermediaries sites including Amazon.

In our set-up, the platform has private information about demand. This modelling option intends to account (in a very simple way) for the digital platforms' ability to collect, gather and process enormous volumes of data $^{4}$ on their customers (e.g. demographic data, data on consumers' online behavior, or data on consumers' previous online searches and purchases). In this respect, Petro (2017) actually argues that "Amazon has a better understanding of the customer than any other retailer. The Motley Fool estimates that over 80 million people are Amazon Prime members. With this data, it is capable of building analytic models which can predict what these consumers will want, how much they will want, and when they will want it." In a completely different set-up, Heiko and Peitz (2017) find that an advertising agency or an Internet portal can make use of a tracking technology which allows it to perfectly identify a consumer's preferred product category, giving support to our assumption that the platform may be better informed about demand than the sellers.

The strategic interaction between the representative seller and the privately informed platform is here examined within a sequential game. In the first stage of this game, the platform first (privately) learns the true value of the good for consumers and then proposes a trading contract to the seller. This contract may be either a marketplace contract (in which all the commercial decisions are transferred to the seller) or a reselling contract (in which the platform itself defines the platform's sales level). In the second stage, after revising her beliefs about the consumers' tastes using the Bayes' Rule, the seller decides to accept or reject the platform's proposition. Finally, in the last stage, the seller takes her sales decisions: in the case of a marketplace contract, she chooses both the level of direct sales and the level of sales intermediated by the platform, whereas in the case of a reselling contract, she only chooses the level of direct sales. In this context, the choice of the platform sales is a "transferable action" ${ }^{5}$ : it is made by the platform under the reselling mode and by the seller under the marketplace mode. Differently, the choice of the direct sales level is

\footnotetext{
3 This modelling option is aligned with the concept of "augmented retail" (corresponding to a blend of digital and physical sale channels), which is becoming more and more prevalent. A number of papers (Brynjolfsson et al., 2009; Goolsbee, 2001; Smith and Telang, 2010; Hilton and Wiley, 2010) investigated the impact of electronic sales on traditional brick-and-mortar sales which is a related but different question (since here we are more interested in looking at the business model of the platform).

${ }^{4}$ According to Marr (2017), "Amazon compares products we browse and buy with millions of other customers around the world. By building a profile of our habits, it is able to match us with products and recommendations from others, which will most likely fit our needs. The Big Data technology at work here is known as a recommendation engine and Amazon's was one of the first, and most sophisticated".

5 We use here the distinction introduced by Hagiu and Wright (2015b).
}

a "non-transferable action": it is determined by the seller in the reselling mode as well as in the marketplace one.

We find that asymmetric information (with the platform being better informed than the seller about final demand) may indeed lead the platform to prefer to resell a high-value good rather than acting as a pure marketplace. More precisely, we find that there is a separating equilibrium in which, all other things equal, the platform is willing to act as a reseller for high demand products but not for low demand products. This separating equilibrium is more likely to occur when: (i) the consumers' willingness to pay for the high-value good is sufficiently larger than their willingness to pay for a low demand product (which opens the door to potentially large information rents); (ii) the negative spillover between the two vending sites (due to some degree of substitution between them) is not too large.

In the literature, it is well established that the marketplace mode allows for the internalization of the spillovers between two vending sites since this business mode transfers to the seller the sales' choice in both channels (direct sales and platform sales), thereby eliminating any double marginalization issues (Tirole, 1988). For this very reason, under complete information, we find that, as expected, the marketplace business model generates, at the same time, greater aggregate profits and greater platform's profits (in comparison with the reselling business model).

When the platform has private information on final demand, the business model choice is no longer exclusively determined by the need to eliminate double marginalization issues. Indeed, the platform shall also consider the possibility of obtaining informational rents (everything else the same). More precisely, the platform faces a trade-off between: (i) letting the informed party (the platform) adjust its own sales level to the state of demand (by proposing a reselling contract), at the cost of not internalizing the externalities between direct sales and platform sales, or (ii) letting the uninformed party (the seller) choose the level of sales in the two sites (by offering a marketplace contract) at the cost of not adapting them to the true state of demand.

We find that the platform will never be interested in reselling a low demand product. In this case, there is not much room for informational rent and therefore it prefers to offer a marketplace contract. Now, what about a high-value good? In the context of our model, the platform may prefer to offer a reselling contract, depending on two parameters: (i) the degree of substitutability between the direct vending site and the platform's vending site and (ii) the ratio between the consumers' willingness to pay for a high-value good and their willingness to pay for a low-value good. When the former is not too important and the latter is large enough, the only Perfect Bayesian Equilibrium satisfying the Cho and Kreps (1987) Intuitive Criterion is a separating equilibrium in which the platform always resells a high quality good. The rational behind this result is the following: the strategic cost of reselling under these circumstances is small (as the spillover between the two vending sites is limited) while the informational benefit is important. When the sites are strong substitutes and/or there is a small gap in the consumers' willingness to pay for a high-value versus a low-value good, the only Perfect Bayesian Equilibrium is a pooling equilibrium where the platform always acts as marketplace for both types of goods (high-value and low-value goods). Finally, for intermediate parameter values, there exist three Perfect Bayesian Equilibrium: a separating one, a pooling one and an hybrid equilibrium where the platform resells the high value good with a certain probability, which is strictly positive and lower than 1.

Related literature. A growing number of papers has investigated the factors explaining why platforms like Amazon act sometimes as resellers and sometimes as a pure marketplace. As far as we know, the only paper which considers the role of interactions 
between direct sales and platform sales is Abhishek et al. (2015). Under complete information, they analyze the platform's choice between the reselling and the marketplace modes. They propose a model in which a good is distributed both through a traditional channel (brick and mortar retailing) and an electronic channel (platform). Under the reselling mode, ${ }^{6}$ the retailer buys the product at a fixed wholesale price and sets the retail price. Under the marketplace mode, the seller is the one choosing the retail price and the platform retains a fixed fraction of her revenues. The basic result is that the reselling mode is preferred to the marketplace mode when electronic sales stimulate traditional sales. Otherwise, the authors find that the platform prefers to work as a marketplace between buyers and sellers.

Jiang et al. (2011) try, as we do here, to explain why retail platforms, like Amazon, resell high-demand products while letting third-party independent sellers sell low-demand products (marketplace mode). They also build a signalling model. However, in their two-period model, it is the seller who has initially private information about demand (high or low). In the first-period, the product is sold directly by the seller, who pays a fixed unit fee to the platform. However, the latter revises its priors on the seller's type from observing the price-quantity pair selected by the independent seller. Anticipating this possibility, the seller may try to hide her type by reducing the level of her first-period services to consumers. Based on its observation, the platform chooses the reselling mode or sticks to the marketplace one in the second period. The authors show that their model has a separating equilibrium (in which the platform identifies the independent seller's type and resells the high-demand good) when theex-ante probability of high demand is above a given threshold. The game has a pooling equilibrium (in which the seller's type is not identified and the platform chooses the marketplace mode) when such probability is below this critical value.

Here, we look at a different case in which the platform is actually better informed than the sellers. Hence, we are looking at a different problem, trying to account for the evidence that Internet giants, such as Amazon, may have "a better understanding of the customer than any other retailer" (Petro, 2017).

Interesting, but more loosely related works, are the papers by Hagiu (2007); Hagiu and Wright (2013, 2015a, 2015b); Hagiu (2007)'s paper is "a first pass" to compare the reselling and the "two-sided platform" modes. It is mostly focused on the analysis of "chicken and egg" problems for two-sided platforms (the more severe this problem is, the more often the reselling mode will be selected by the platform). Hagiu (2007) also surveys a lot of other factors affecting the platforms' business modus operandi (such as the complementarity or substitutability between sellers' products that are not internalized in the prices, indirect network effects between buyers and sellers, as well as asymmetric information between sellers and the platform). However, this work "rulesout direct sales by sellers to consumers", ${ }^{7}$ which constitute a key element in our set-up.

The main interest of Hagiu and Wright (2015a) is to model the choice between the reseller and the marketplace modes "as a decision between whether control rights over a non-contractible decision variable (the choice of some marketing activity) are better held by suppliers (in the marketplace mode) or by the intermediary (in the reseller mode)". This is similar to our paper in which, however, the non-contractible decision variable is the level of direct seller's sales (instead of a marketing activity). In their approach, the choice leans toward the reselling mode (resp. the marketplace mode) when the platform (resp. the seller) is better in-

\footnotetext{
${ }^{6}$ It is assumed that the traditional channel always uses it.

${ }^{7}$ p.117
}

formed about "the optimal tailoring of marketing activities". As in Hagiu (2007), network effects between sellers and unfavorable expectations are shown to tilt the choice toward the reselling mode.

Hagiu and Wright (2015b) generalize the previous paper by analyzing the factors which favor the adoption of the multi-sided platform (MSP) mode versus vertical integration (VI) or the reseller mode. They focus on the first trade-off (MSP vs VI) while referring to Hagiu and Wright (2015a) for the second one.

Even, more loosely related are the works of Balasubramanian (1998) or Yoo and Lee (2011). Those authors deal with the introduction of an Internet distribution channel in a market where there already existed a traditional physical channel (brick-and-mortar). They highlight the important role played by the different features of the Internet channel vis-á-vis the traditional vending site. In particular, the authors are more interested in understanding the impact of introducing the new online channel rather than looking at the platforms' business choice.

Finally, notice also a paper by Foros et al. (2017) who study the marketplace model adopted by Apple and Google. ${ }^{8}$ They set up a single period model of perfect and symmetric information with imperfect competition both at the upstream (sellers) and the downstream (platform) level, investigating the optimal pricing strategies for the platforms and the sellers. They prove that "the agency ${ }^{9}$ model may not be universally adopted even if adoption would mean higher profits for all firms."

The rest of the paper is organized as follows. In Section 2, we set up the model. In Section 3, we study the full information benchmark. We examine the Perfect Bayesian Equilibria of our game in Section 4, providing an analytical characterization for the Least Cost Separating Equilibrium, in which the platform chooses to resell only high-value goods, preferring to work as a marketplace for low-demand goods. Section 5 concludes the paper.

\section{The model}

A good is produced by a given seller, who may sell the good directly to consumers and/or via a platform. The latter may act as a reseller who buys the product from the seller and resells it to final consumers (i.e. a pure reseller) or as a simple marketplace. In the former case, the platform fixes the quantities it sells itself while the seller determines the level of her direct sales, after observing the platform's sales decisions (which are known to the seller as we assume that the platform sells all the units of the good it buys from the seller). In the marketplace case, the seller is in charge of choosing her sales, both in the direct vending site and in the marketplace (i.e. the sales mediated by the platform are decided by the seller). For the sake of simplicity, and without loss of generality, we normalize unit production and distribution costs to zero.

In each distribution channel, the following inverse demand functions are assumed:

$p=\theta-y-\delta x$,

$v=\theta-x-\delta y$,

where $p$ denotes the price of the good when it is sold through the platform; $v$ is the price of the good charged by the seller when she directly sells her good to the final consumers; $\theta$ is the consumers' maximum willingness to pay for the good (or, equivalently, a measure of the width of the potential market) and $\delta \in(0,1)$ is

\footnotetext{
${ }^{8}$ For example, the retail price of the popular game Angry Birds is controlled by its inventor, Rovio Entertainment, and e-book retail prices are determined by the publishers. Apple and Google keep $30 \%$ of the revenue created when a sale is made through its platform.

9 The agency model mentioned here refers to what we call "Marketplace Model" in the present paper.
} 
the parameter that formally measures the degree of substitutability between direct sales $x$ and platform sales $y$.

The demand specification is based on the underlying assumption that opening a second vending site allows to increase the aggregate sales of the good (measured at an identical price). However, the aggregate sales, when the second distribution channel is used, are never more than twice the sales at a single site. ${ }^{10}$ According to (1), at identical selling prices, the ratio between aggregate sales when the two distribution sites are used and the sales when only one vending site is active is ${ }^{11}$ equal to $\frac{2}{1+\delta}$, which, as expected, is decreasing in $\delta$.

In our set-up, $\theta$ is assumed to be private information of the platform. The seller does not know the true value of $\theta$. Her priors are such that $\theta=\theta_{H}$ (the product is a high-value one) with probability $\mu$ and $\theta=\theta_{L}$ (the product is a low-value one) with probability $1-\mu$. We denote by $k=\frac{\theta_{H}}{\theta_{L}}$ the ratio between the consumers' maximum willingness to pay for a high-value good and the consumers' maximum willingness to pay for a low-value good. Hence, $k$ provides information on the demand expansion when the product is a high-value rather than a low-value one. This parameter will play an important role in our analysis since it allows us to assess whether there is a large potential for information rents (which amounts to a large $k$-value).

The strategic interaction between the seller and the platform is examined within a sequential game with asymmetric information, whose timing is as follows:

1. The platform privately learns whether the good is a high-value good $\left(\theta=\theta_{H}\right)$ or a low-value one $\left(\theta=\theta_{L}\right)$;

2. The platform decides its business model strategy $(S)$, proposing to the seller either a reselling contract $(S=R)$ or a marketplace contract $(S=M)$. A reselling contract specifies the level of platform sales $y_{X}$ and a transfer $T_{X}$ to the seller. In the case of a marketplace contract, the platform only defines the fixed fee $F$ that it charges the seller in order to give her access to the platform (with all decisions on the level of platform sales being made by the seller, afterwards);

3. The seller observes the contract offered by the platform and revises her beliefs using Bayes' Rule. Then, she decides whether to accept or reject the platform's proposition.

4. If the seller has accepted a reselling proposition, given her expost beliefs about $\theta$, she selects a level $x$ of direct sales as a best reply to $y_{X}$. If, instead, she has accepted a marketplace contract, at this stage, the seller simultaneously chooses the level of direct sales and the level of platform sales $(x$ and $y$, respectively) in order to maximize her expected profits, given her expost beliefs about $\theta$.

In order to find the equilibrium of the model, we proceed by backwards induction, first solving the last stage of the game and then finding the solution of the previous stages.

\section{The full information benchmark}

Let us first analyze the full information benchmark. Given the structure of our game, we need to separately examine two different subgames, defined on the basis of the platforms' business model choice: the marketplace subgame; and the reselling subgame.

\footnotetext{
10 The basic assumption is that, at any given price, the aggregate sales of the two vending sites (when they are both active) are greater than the sum of their sales when each one of them is the only distribution channel. For the sake of simplicity, we suppose that the two sites are symmetrical. A generalization is available from the authors upon demand.

${ }^{11}$ Let $p=v$. If only one site is open, the direct demand for the good is $\theta-p$. When the two sites are open, the aggregate direct demand is $\frac{2}{1+\delta}(\theta-p)$. Thus, opening a second site expands demand by a coefficient $\frac{2}{1+\delta}$.
}

\subsection{Marketplace}

Under a marketplace contract, the seller, who knows the realized value of the good, $\theta_{X}, X=H, L$, chooses the level of sales at both sites (platform and direct sales) in order to maximize her profits:

$\max _{x, y}\left[x\left(\theta_{X}-x-\delta y\right)+y\left(\theta_{X}-y-\delta x\right)-F\right]$.

The equilibrium level of direct and platform sales are given by

$y^{A}=x^{A}=\frac{\theta_{X}}{2(1+\delta)}$,

and aggregate profits are:

$\frac{\theta_{X}^{2}}{2(1+\delta)}$.

Hence, the platform will set the transfer $F$ in such a way that, for the selected transfer $F^{a}$, the seller's participation constraint is binding. Put otherwise, the seller's equilibrium profits should not be smaller than the level of profits obtained when she only uses its direct distribution channel to sell the good. Hence, to obtain the seller's stand-alone profit, we need to look for the solution of the seller's profit maximization problem w.r.t $x$, when only the direct sales channel is active. This yields to a level of equilibrium (direct) sales equal to $\frac{\theta_{X}}{2}$, with the corresponding stand-alone profit being $\frac{\theta_{X}^{2}}{4}$.

Hence,

$F^{a}=\frac{\theta_{X}^{2}}{2(1+\delta)}-\frac{\theta_{X}^{2}}{4}=\frac{(1-\delta)}{4(1+\delta)} \theta_{X}^{2}$.

Summing up, in a full information with a marketplace contract, the seller's profit is $\frac{\theta_{X}^{2}}{4}$, whereas the platform's profit is equal to the fixed fee $F^{a}$. Let us now look at the reselling subgame.

\subsection{Reselling}

In the reselling contract, the seller chooses the direct sales level $x$ in order to maximize her profits

$\max _{x}\left[x\left(\theta_{X}-x-\delta y\right)+T\right]$

given the level $y$ of platform's sales and the fixed transfer $T$ received from the platform.

Thus, when solving the seller's profit maximization problem, one obtains the following best reply function

$x\left(\theta_{X}, y\right)=\max \left\{\frac{\theta_{X}-\delta y}{2}, 0\right\}$,

defining the optimal level of direct sales $(x)$, as a function of the platform's sales levels (and the demand parameter $\theta_{X}$, which under full information is also known by the seller).

The platform acts like a Stackelberg leader, maximizing its profit

$\max _{y}\left[y\left(\theta_{X}-y-\delta x\left(\theta_{X}, y\right)\right)-T\right]$

subject to the seller's participation constraint. Again, this constraint simply requires that the seller's profit should at least equal to her stand-alone profit, $\frac{\theta_{X}^{2}}{4}$, which is obtained when the seller rejects the contract proposal ${ }^{12}$, opting for not doing any sales through the platform, i.e;

$$
\left(x\left(\theta_{X}, y\right)\right)^{2}+T \geq \frac{\theta_{X}^{2}}{4} .
$$

\footnotetext{
12 In which case $y=0$.
} 
Since the platform benefits from choosing $T$ as low as possible, $y$ is then set to maximize the following function (which already incorporates the seller's participation constraint).

$y\left(\theta_{X}-y-\delta \frac{\theta_{X}-\delta y}{2}\right)+\left(x\left(\theta_{X}, y\right)\right)^{2}-\frac{\theta_{X}^{2}}{4}$.

Accordingly, in the reselling subgame, the platform's full information sales $\left(y_{X}^{f}\right)$, transfer $\left(T_{X}^{f}\right)$ and payoff $\left(\Pi_{X}^{f}\right)$ are given by ${ }^{13}$ :

$$
\begin{aligned}
y_{X}^{f} & =\frac{2(1-\delta) \theta_{X}}{4-3 \delta^{2}}, \\
T_{X}^{f} & =\frac{\delta\left(4-\delta+\delta^{2}\right) \theta_{X}^{2}}{\left(4+\delta^{2}\right)^{2}}, \\
\Pi_{X}^{f} & =\frac{\theta_{X}^{2}(1-\delta)^{2}}{4-3 \delta^{2}} .
\end{aligned}
$$

In order to solve the full game, we now need to compare the platform's equilibrium profit under a marketplace contract and a reselling contract.

\subsection{Comparison of the platform's strategies under full information}

It is illuminating to compare the full information platform's profits when it selects the marketplace business model versus the reselling contract. The respective profits are given by (3) in the former case and by the last equation in (5) in the reselling one. The difference is

$$
\frac{\theta_{X}^{2}(1-\delta)}{4(1+\delta)}-\frac{\theta_{X}^{2}(1-\delta)^{2}}{4-3 \delta^{2}}=\theta_{X}^{2} \frac{(1-\delta) \delta^{2}}{4(1+\delta)\left(4-3 \delta^{2}\right)} .
$$

This difference equals 0 when $\delta=0$ (no externality between the two vending sites) and when $\delta=1$ (sales at the two sites are perfect substitutes so that selling at the platform does not bring any additional profits, whatever the distribution mode). It is strictly positive for all other values of $\delta$, showing that the marketplace business model gives higher profits to the platform (in comparison to the reselling business model). The marketplace model also necessarily yields greater aggregate profits than the reselling model, since in both cases the seller's profit is equal to $\frac{\theta_{X}^{2}}{4}$, which corresponds to the maximum profit from direct sales only.

The rationale behind the superiority of the marketplace mode under full information is straightforward. In the context of a marketplace model, we have that the same agent, the seller, chooses the level of direct sales as well as the level of platform sales. Hence, when doing so, the seller internalizes all the externalities between the two vending sites. In contrast, in the reselling business strategy, the seller only maximizes her profits from direct sales, ignoring the external effects of the direct sales on the platform's profits. It's only when there are no externalities $(\delta=0)$ or when selling through the platform does not possibly increase aggregate profits $(\delta=1)$, that the two modes are equivalent.

We now turn our attention to the model with asymmetric information, which allows us to show that, all other things equal, the platform may no longer always prefer to propose a marketplace business model to the seller.

\section{Perfect Bayesian equilibria}

In a full information set-up, the possibility to internalize the externalities between the two sales channels always gives a strategic advantage to the marketplace model over the reselling contract. When, as we suppose here, the platform has private information about the true demand state (high or low), this may no longer

\footnotetext{
${ }^{13}$ It is easy to check that $x\left(\theta_{X}, y_{X}\right)>0$.
}

be the case. In particular, the platform may actually prefer the reselling mode over the marketplace model to obtain greater informational rents. This is more likely to occur when the consumers' willingness to pay for the good in the high-demand scenario is sufficiently high (leading to potentially large informational rents). The platform's business model choice may signal the true demand state and therefore, we examine the strategic interaction between the platform and the seller within a signalling game framework.

We start by proving an important result, which shows that reselling a low-value good is always a dominated business strategy for the platform.

Proposition 1. In equilibrium, the platform never resells a low-value good.

Proof. If the platform were to resell the low-value good, its maximum possible profits would be equal to

$$
\begin{aligned}
& \max _{y} y\left(\theta_{L}-y-\delta \frac{\theta^{e}-\delta y}{2}\right)+\left(x\left(\theta^{e}, y\right)\right)^{2}-\left(\frac{\theta^{e}}{2}\right)^{2} \\
& =\frac{\left(\theta_{L}-\delta \theta^{e}\right)^{2}}{4-3 \delta^{2}}
\end{aligned}
$$

where $\theta^{e}=\rho \theta_{H}+(1-\rho) \theta_{L}, \rho$ being the ex-post probability that the good is a high-value one (conditional on the platform's choosing a reselling contract, $R$ ). Since a marketplace mode would, in the low-demand scenario, guarantee to the platform profits at least equal to $\frac{(1-\delta) \theta_{L}^{2}}{4(1+\delta)}>\frac{\left(\theta_{L}-\delta \theta^{e}\right)^{2}}{4-3 \delta^{2}}$, it follows that the platform will always prefer to act as a marketplace when the true state of $\theta$ is $\theta_{L} \cdot$

From Proposition 1 follows that indeed the platform's business model choice may be a signal of the true demand state. In particular, if the platform chooses the reselling mode, the seller immediately concludes that the good is a high value $\left(\theta=\theta_{H}\right)$ one:

$\rho\left(\theta=\theta_{H} \mid S=R\right)=1$.

Now, what about the probability that the consumers' willingness to pay for the good is high when the platform chooses the marketplace mode? Given (7), when the $H$-type platform chooses $R$ with probability $\lambda \in[0,1]$ and $M$ with complementary probability, the Bayes' Rule implies that the ex-post probability that the product is a high value one conditional on the choice of a marketplace system must be

$\rho\left(\theta=\theta_{H} \mid S=M\right)=\frac{\mu(1-\lambda)}{\mu(1-\lambda)+(1-\mu)}$.

Notice that, when $\lambda=1, \rho\left(\theta=\theta_{H} \mid S=M\right)=0$ and, when $\lambda=$ $0, \rho\left(\theta=\theta_{H} \mid S=M\right)=\mu$. For any value $\lambda \in[0,1], \rho\left(\theta=\theta_{H} \mid S=\right.$ M) $\in[0, \mu]$.

It is well-known that there may be three possible types of equilibria of a signalling game: separating equilibria, pooling equilibria and hybrid equilibria. In the paper, we focus on the separating equilibria, in which the platform's business model actually depends on the true demand state: in the case of low-demand products, the platform always acts as a marketplace, whereas in the case of high-demand goods, the platform opts for a reselling strategy. As, will be shown below, the separating equilibria will emerge when $k$ is large enough, meaning that the consumers' willingness to pay for a high-value good is sufficiently higher than their willingness to pay for a low-value good (i.e. the platform's informational rents are potentially high).

In what follows, we provide an analytical characterization of the separating equilibria. The analysis of the hybrid and the pooling equilibria is left to the Appendix. 


\subsection{Separating equilibria}

In a separating equilibrium, the platform chooses different business trading strategies when the product is a high-value or a lowvalue one. The seller' beliefs are then revised accordingly, so that the seller is actually able to learn the true state of demand i.e. $\theta_{H}$ or $\theta_{L}$.

From Proposition 1, there cannot exist an equilibrium in which the platform would resell the low value good and distribute the high value one as a marketplace. Thus, the only possible separating equilibria are such that:

- Upon observing privately that the product is a low value one, the platform chooses to act as a marketplace, i.e. $S(L)=M$, and asks $F_{L}$ from the seller;

- Upon observing privately that the product is a high value one, it chooses to act as a reseller, i.e. $S(H)=R$, or, equivalently, $\lambda=1$, selling quantities $y_{H}$ and paying $T_{H}$ to the seller.

From (8), upon observing that the platform chooses a marketplace mode, the seller infers that the product is low value one, i.e. $\rho\left(\theta=\theta_{H} \mid S=M\right)=0$.

At equilibrium, the $H$-type platform should not benefit from pretending that the product is a low value one, leading to the following condition:

$$
\begin{aligned}
& {\left[\left(\theta_{H}-y_{H}-\delta \frac{\theta_{H}-\delta y_{H}}{2}\right) y_{H}\right]-\left[\frac{\theta_{H}^{2}}{4}-\left(\frac{\theta_{H}-\delta y_{H}}{2}\right)^{2}\right] \geq F_{L}} \\
& \quad=\frac{(1-\delta) \theta_{L}^{2}}{4(1+\delta)} .
\end{aligned}
$$

The LHS of (9) equals the platform's (direct) operating profits when selling a quantity $y_{H}$ minus the price $T_{H}$ it pays to its supplier to guarantee her a profit just equal to its stand-alone value. ${ }^{14}$ The RHS is the platform's profit when it pretends that the product is a low-value one. It just equals to the maximum transfer which it can ask in that case from the seller, who, upon observing $S=M$, would anticipate that consumers' willingness to pay is low (since we are looking here at a separating equilibrium).

Notice that Proposition 1 guarantees that the $L$-type platform never benefits from choosing a business strategy $R$, which would amount to pretend that the product is a high-value one. Accordingly, the $L$-type platform's incentive constraint is automatically satisfied.

When there exist several separating equilibria, i.e. several values of $y_{H}$ which satisfy the incentive constraints (9), ${ }^{15}$ it is natural to focus on the ones which maximize the H-type platform's profits, i.e. the Least Cost Separating Equilibria (LCSE).

Proposition 2. A Separating Equilibrium, in which the platform resells the good when $\theta=\theta_{H}$ and distributes it as a marketplace when $\theta=\theta_{L}$, exists iff $k \geq \frac{1}{G}=\sqrt{\frac{4-3 \delta^{2}}{4\left(1-\delta^{2}\right)}}$.

Proof. Since the type L-platform's incentive constraint is always satisfied and the participation constraint is satisfied by construction, there exists a separating equilibrium iff the type H-platform's incentive constraint (9) is satisfied. This is obviously the case iff the maximum of the LHS of (9) w.r.t $y_{H}$, i.e. $\frac{(1-\delta)^{2} \theta_{H}^{2}}{4-3 \delta^{2}}$ is not smaller than $\frac{(1-\delta)^{2} \theta_{L}^{2}}{4\left(1-\delta^{2}\right)}$, i.e. iff

$k \geq \sqrt{\frac{4-3 \delta^{2}}{4\left(1-\delta^{2}\right)}}$.

\footnotetext{
${ }^{14} T_{H}$ is the minimum price which satisfies the supplier's participation constraint.

15 It is implicitly assumed that the participation constraints are satisfied.
}

Notice that the threshold $\sqrt{\frac{4-3 \delta^{2}}{4\left(1-\delta^{2}\right)}}$ in condition (10) corresponds to the square root of the ratio between marketplace and reselling platform's full information profits, for any $\theta$. This threshold is greater than 1 for all $\delta \neq 0$ and it is increasing in $\delta$.

Condition (10) simply states that the ratio between the consumers' maximum willingness to pay under different realizations of the demand parameter $\left(k=\frac{\theta_{H}}{\theta_{L}}\right)$ must be greater than the square root of the ratio between the marketplace and the reselling platform's full information profits, for a given $\theta$. The LHS $(k)$ provides a measure of the platform's informational rents on good demand states, ${ }^{16}$ whereas the RHS provides a measure of the strategic advantage of marketplace over reselling. ${ }^{17}$

Hence, the results of Proposition 2 are quite consistent with intuition. The existence of a (least-cost) separating equilibrium in which a low-value good is distributed via a marketplace while a high-value good is resold by the platform is more likely when $k$ is large and when the externalities between the two sites, as represented by $\delta^{2}$, are small enough. Indeed, the smaller the externalities between the sites, the less important the strategic advantage of a marketplace mode (which allows the seller to internalize the externalities between the two vending sites) over a reselling contract (in which decisions on direct sales and platform sales are taken by different firms).

Corollary 1. In the LCSE, when $\theta=\theta_{H}$, the platform: (i) optimally resells the quantity $y_{H}^{f}=\frac{2(1-\delta) \theta_{H}}{4-3 \delta^{2}}$; and (ii) it pays the transfer $T_{H}=$ $\frac{\theta_{H}^{2}}{4}-\left(\frac{\theta_{H}-\delta y_{H}^{f}}{2}\right)^{2}$ to the seller.

Proof. The best separating equilibrium from the H-platform's point of view, i.e. the LCSE, is the one which maximizes its profits, with $\left\{y_{H}^{f}=\frac{2(1-\delta) \theta_{H}}{4-3 \delta^{2}}, T_{H}=\frac{\theta_{H}^{2}}{4}-\left(\frac{\theta_{H}-\delta y_{H}^{f}}{2}\right)^{2}\right\}$.

Corollary 1 provides a full description of the LCSE. It shows that, in this equilibrium, the level of platform sales with asymmetric information actually coincides with the level of platform sales in a full information scenario (with $\theta=\theta_{H}$ ). However, in this case, the platform takes advantage on its private information, to pay a lower fee to the seller, i.e., $T_{H}<T_{X}^{f}$.

The separating equilibria studied above are not the only equilibria in our signalling game. As shown in Appendix, the game also displays pooling and hybrid equilibria. In a pooling equilibrium, the platform's business model is not affected by the true realization of demand. From Proposition 1, the only possible pooling equilibrium is such that $S(H)=S(L)=M$ and $\rho(X=H \mid S=M)=\mu$. In this equilibrium, the platform chooses the marketplace system whatever the value of the good and therefore the seller does not update her beliefs upon observing the marketplace proposal.

In a hybrid equilibrium the $L$-type platform offers a marketplace contract, while an $H$-type platform randomizes, i.e, it chooses $R$ with probability $\lambda$ and $M$ with complementary probability. According to the Bayes' Rule, the ex-post probability that the product is a high value one conditional on the choice of a marketplace system is then given by (8). The next subsection analyses in more detail the equilibrium configuration in the $(\delta, k)$-space, leaving to the Appendix the analytical characterization of the pooling and the hybrid equilibria.

\footnotetext{
16 The informational advantage of the platform under reselling is the greater, the higher is the ratio between the consumers' willingness to pay for the high and the low-value good: it is precisely measured by the ratio $\frac{\theta_{H}}{\theta_{L}}=k$.

17 This advantage is precisely measured by the ratio between the full information profits of the two business strategies, $\frac{1}{G}$.
} 


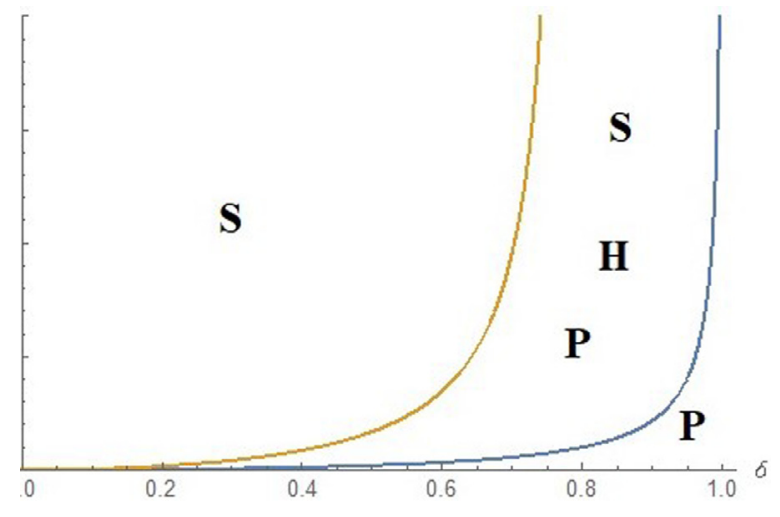

Fig. 1. Equilibrium configuration in the $(\delta, k)$-space.

\subsection{Equilibrium configuration in the $(\delta, \mathrm{k})$-space}

The common point between the three possible types of equilibria is that the platform always prefers to use the marketplace mode to distribute a low value good. At a separating equilibrium, it always chooses the reselling mode to distribute a high value good. At an hybrid equilibrium, it randomizes between marketplace and reselling. At a pooling equilibrium, it distributes it as a marketplace.

The Fig. 1 below (which is drawn for $\mu=0.845$ ) pictures the equilibrium configuration in the $(\delta, k)$-space. ${ }^{18}$ There are three areas:

- $S$, where the unique equilibrium that meets (Cho and Kreps, 1987) Intuitive Criterion is the least cost separating equilibrium;

- $P$, where the pooling equilibrium is the unique Perfect Bayesian Equilibrium (PBE for short);

- SHP, where the three equilibria exist: separating, pooling and hybrid.

As Fig. 1 shows, in the area $S$, the only PBE meeting the Intuitive Criterion is the (least cost) separating equilibrium, where the high value good is distributed by the platform under a reselling contract, whereas the low-value good is distributed within a marketplace system. This equilibrium occurs when (i) the externalities between the vending sites are weak, (ii) the ratio between the willingness to pay for the high-value and for the low-value goods is high and (iii) the ex-ante probability of a high-value good is small enough.

In contrast, in the area $P$, the only PBE meeting the Intuitive Criterion implies a pooling strategy, for which the platform prefers to distribute both types of goods as a marketplace. In this subdomain, the externalities between direct sales and platform sales are large ( $\delta$ is large) and/or the difference in consumers' willingness to pay for the two demand realizations is not too large (i.e. $k$ is small enough). On the one hand, the strong externalities favor the marketplace system (which allows for the internalization of those externalities by assuring that the same player takes decisions on the level of direct sales and the level of platform sales). On the other hand, the small difference between the goods' value limits the informational advantage of using a reselling strategy.

Finally, for the subdomain of parameters in the area SHP, three possible PBE exist: pooling, separating and hybrid. In this area, both externalities between the two vending sites $(\delta)$ and the ratio between $\theta_{H}$ and $\theta_{L}$ are relatively mild. The intuition behind this equilibrium multiplicity lies on the existence of a self-reinforcing mechanism which makes the hybrid equilibrium look like an un-

\footnotetext{
${ }^{18}$ Remember that $\delta$ is the degree of substitutability between the two sites and $k$ the ratio between the willingness to pay for the high and the low-value good.
}

stable equilibrium and the separating as well as the pooling equilibria look like (locally) stable ones. Indeed, when the frequency with which the platform chooses to resell the $H$-good $(\lambda)$ increases, the Bayes' Rule (8) leads to a reduction in the ex-post probability that $\theta=\theta_{H}$, conditional on the platform having chosen a marketplace mode. This way, the platform's marketplace profits, ${ }^{19}$ go down, thus increasing the relative profitability of Reselling versus Marketplace. On the contrary, any reduction in $\lambda$ leads to a reduction of the relative profitability of a marketplace contract vis-à-vis a reselling contract.

\section{Conclusion}

In this paper, we investigate optimal managerial strategies in markets where product sellers may distribute their goods using two distinct channels: direct vending sites and intermediation platforms, which are not necessarily perfect substitute channels. We assume that, contrary to the level of direct sales, the level of the platform's sales may be a transferable decision of the seller to the platform, who has private information on the true demand realization.

We propose to analyze the strategic interaction between the seller and the platform within a sequential signalling game. Everything else the same, this stylized model allows us to examine how the platform's optimal business choice (marketplace mode versus reselling mode) may be affected by the market features such as the degree of substitutability between direct sales and platform sales; or the consumers' additional willingness to pay for a high-value good (vis-à-vis their willingness to pay for a low-demand good).

While it is clearly the case that, under full information, the marketplace mode leads to the highest-profits for all the market players (the marketplace mode allows for the internalization of all the profit effects originated by the intermediated sales), in an imperfect information set-up, that may not necessarily be the case.

Accordingly, our results provide one possible explanation (not excluding others) for the observation that the platform tends to resell more high-value goods than low-value ones. This finding is consistent with previous empirical evidence pointed by Jiang et al. (2011) on the distribution strategy of important digital platforms such as Amazon. It is also aligned with the spirit of the Chief Executive Jeff Bezos's bons mots"Your margin is my opportunity". ${ }^{20}$

Given that the platform never benefits from reselling a lowvalue good, the adoption of the reselling mode signals that the good is a high-value one. A separating equilibrium, in which the platform always resells the high-value good, occurs when the two vending sites are not strong substitutes and/or when the highvalue good is substantially more valued than the low-value one. A pooling equilibrium, in which the platform always acts as a marketplace, arises in the opposite case. Finally, an area of multiple equilibria (separating, pooling and hybrid) exists for intermediate parameters values.

In the future, several interesting extensions of this model may be analyzed. In particular, it may be interesting to extend the present approach to account for competition between platforms and for the existence of several sellers, incorporating network effects. Another interesting extension would be to look at the platform's incentives to act in the upstream market as well (as a producer), in line with the recent developments in the strategy of firms like Amazon. Indeed, this platform has recently bought "Whole Foods", it is already involved in media content production (e.g. the movie "Manchester by the Sea") and it also produces several private brand products.

\footnotetext{
19 As given by expressions (11) and (12) in the Appendix

20 Citation from an article in the Wall Street Journal, written by Mims (2018).
} 


\section{Appendix A. Pooling equilibria}

In a pooling equilibria, the platform chooses the same strategy regardless of the state of demand. Proposition 1 allows us to pin down out-of-equilibrium beliefs in order to evaluate possible deviations. Indeed, as Proposition 1 establishes that the choice of a reselling strategy when the good is a low value one is always a dominated strategy, we can naturally suppose that the seller, upon observing a deviation towards the reselling mode, must conclude that the good is a high value one. The pooling equilibrium then satisfies the Cho and Kreps (1987) "Intuitive Criterion" iff the $H$ type platform does not benefit from choosing the reselling mode (thus revealing its type to the seller).

Lemma 1. A pooling equilibrium, in which the platform always chooses the marketplace mode satisfies (Cho and Kreps, 1987) "Intuitive Criterion" if either:

$$
\begin{aligned}
& \text { (i) } \mu \geq G=\sqrt{\frac{4-4 \delta^{2}}{4-3 \delta^{2}}} \text { or } \\
& \text { (ii) } \left.\mu<G \text { and } k \in] 1, \frac{1-\mu}{G-\mu}\right] \text {. }
\end{aligned}
$$

Proof. The Intuitive Criterion requires that there should be no feasible deviation from the marketplace equilibrium yielding greater profits to the $H$-type platform, when we account for the fact that the platform is automatically recognized as a $H$-type platform when it deviates from marketplace to reselling, since $S=$ $R$ is a dominated strategy for a $L$-type platform as a result of Proposition 1 and expression (7).

The H-type platform's profits, when recognized as such upon deviating, are equal to its full information profits. They should not be greater than its profits at the pooling equilibrium, i.e.

$$
\frac{(1-\delta)^{2} \theta_{H}^{2}}{4-3 \delta^{2}} \leq \frac{(1-\delta) \bar{\theta}^{2}}{4(1+\delta)} \text {, where } \bar{\theta}=\mu \theta_{H}+(1-\mu) \theta_{L} \text {. }
$$

This condition holds always true $\forall k>1$ when $\mu \geq G=$ $\sqrt{\frac{4\left(1-\delta^{2}\right)}{4-3 \delta^{2}}}$. When $\mu<G$, the inequality above only holds for $1<k<$ $\frac{1-\mu}{G-\mu}$. Since $\frac{1-\mu}{G-\mu}>\frac{1}{G}$, the Intuitive Criterion eliminates all pooling equilibria which do not satisfy these conditions.

\section{Appendix B. Hybrid equilibria}

Lemma 2. There exists an hybrid equilibrium when either $k \in\left[\frac{1}{G}, \frac{1-\mu}{G-\mu}\right]$ and $G>\mu$,

or,

$k \geq \frac{1}{G}$ and $G \leq \mu$.

In this hybrid equilibrium:

(i) with probability $\lambda=\frac{\mu-\rho}{\mu(1-\rho)}$, where $\rho$ is given by (14), the platform opts for a reselling strategy, setting in that case $y_{H}^{f}=$ $\frac{2(1-\delta) \theta_{H}}{4-3 \delta^{2}}$; with the complementary probability, the platform opts for the marketplace business model,

(ii) When the platform privately knows that the good is a low value one, it always prefers to act as a marketplace.

Proof. When $\theta=\theta_{H}$ and the platform chooses to act as a reseller, it obtains profits equal to $\frac{(1-\delta)^{2} \theta_{H}^{2}}{4-3 \delta^{2}}$. Differently, when $\theta=\theta_{H}$ and the platform decides to become a marketplace, it obtains profits equal to

$\frac{(1-\delta) \widehat{\theta}^{2}}{4(1+\delta)}$ where

$$
\widehat{\theta}=\theta_{H} \frac{\mu(1-\lambda)}{\mu(1-\lambda)+(1-\mu)}+\theta_{L} \frac{(1-\mu)}{\mu(1-\lambda)+(1-\mu)} .
$$

For the type $H$-platform to have interest in randomizing its strategies, the expected profit under a marketplace contract and the expected profit under a reselling contract must be the same. Then, the following necessary conditions for the existence of an hybrid equilibrium follow:

$\lambda=\frac{\mu-\rho}{\mu(1-\rho)}$

with

$\rho=\frac{k G-1}{k-1}$

and $G=\sqrt{\frac{4\left(1-\delta^{2}\right)}{4-3 \delta^{2}}}<1$.

For this to be an equilibrium, it must be that $\lambda \in[0,1]$ and $\rho \in[0, \mu]$. Accordingly, necessary conditions for the existence of an hybrid PBE are:

(i) $k \geq \frac{1}{G}>1$ which guarantees that $\rho \geq 0$;

(ii) $k \leq \frac{1-\mu}{G-\mu}$ which guarantees that $\rho \leq \mu$ when $G>\mu$.

When $G<\mu$, we check that $\rho \leq \mu$ for all $k>1$.

\section{References}

Abhishek, K., Jerath, K., Zhang, J., 2015. To platform-sell or resell? Channel structures in electronic retailing. Manag. Sci. 62, 2259-2280.

Anderson, C., 2006. The Long Tail why the Future of Business is Selling Less of More, Hyperion.

Balasubramanian, S., 1998. Mail versus mall: a strategic analysis of competition between direct marketers and conventional retailers. Mark. Sci. 17, 181195.

Brynjolfsson, E., Hu, Y., Rahman, M.S., 2009. Battle of the retail channels: how product selection and geography drive cross-channel competition. Manag. Sci. 55 (11), 1755-1765

Cho, I.-K., Kreps, D.M., 1987. Signaling games and stable equilibria. Quart. J. Econ. 102, 179-221.

Foros, O., Kind, H.J., Shaffer, G., 2017. Apple's agency model and the role of most-favored-nation clauses. RAND J. Econ. 48 (3), 673-703.

Goolsbee, A., 2001. Competition in the computer industry: online versus retail. J. Ind. Econ. 49 (4), 487-499.

Hagiu, A., 2007. Merchant or two-sided platform. Rev. Netw. Econ. 6 (2), 115-133.

Hagiu, A., Wright, J., 2013. Do you really want to be an Ebay? Harv. Bus. Rev. 91 (3) 102-108.

Hagiu, A., Wright, J., 2015a. Marketplace or reseller? Manag. Sci. 61, 184-203.

Hagiu, A., Wright, J., 2015b. Multi-sided platforms. Int. J. Ind. Org. 43, 162-174.

Heiko, K., Peitz, M., 2017. De-targeting: advertising an assortment of products to loss-averse consumers. Eur. Econ. Rev. 95, 103-124.

Hilton, J., Wiley, D., 2010. The short-term influence of free digital versions of books on print sales. J. Electron. Publ. 13 (1). [online]. URL: http://hdl.handle.net/2027 spo.3336451.0013.101

Jiang, B., Jerath, K., Srinavasan, K., 2011. Firm strategies in the 'mid tail' of platform based retailing. Mark. Sci. 30, 757-775.

Marr, B., 2017. Want to use big data? Why not start via google, facebook, amazon, (etc.). Forbes [online]. [Access date: 28 january 2018]. Url: https://www. forbes.com/sites/bernardmarr/2017/08/14/want-to-use-big-data-why-not-startvia-google-facebook-amazon-etc/2/\#642cb35a5e07.

Mims, C., 2018. The limits of amazon. Wall Street J.. [online]. [Access date: 28 january 2018]. Url: https://www.wsj.com/articles/the-limits-of-amazon1514808002

Petro, G., 2017. Amazon's acquisition of whole foods is about two things: data and product. Forbes [online]. [Access date: 28 january 2018]. Url: https://www. forbes.com/sites/gregpetro/2017/08/02/amazons-acquisition-of-whole-foods-isabout-two-things-data-and-product/\#26d1959ba808.

Rysman, M., 2009. The economics of two-sided markets. J. Econ. Perspect. 23 (3), 125-143.

Smith, M.D., Telang, R., 2010. Piracy or promotion? The impact of broadband internet penetration on DVD sales. Inf. Econ. Policy Spec. Issue 22 (4), 289298.

Yoo, W.S., Lee, E., 2011. Internet channel entry: a strategic analysis of mixed channel structures. Mark. Sci. 30, 29-41.

Zhu, F., Liu, A., 2016. Competing with complementors: an emprical look at amazon.com. Harvard Business School Working Paper 15-044. 